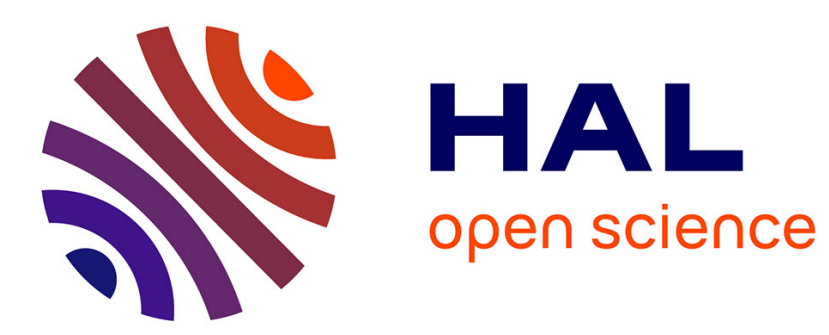

\title{
Intervenir en EPS pour développer l'entretien de soi. Une étude de cas d'un enseignant expérimenté qui débute
}

Denis Loizon

\section{To cite this version:}

Denis Loizon. Intervenir en EPS pour développer l'entretien de soi. Une étude de cas d'un enseignant expérimenté qui débute. Carrefours de l'éducation, 2015, 40. halshs-01240093

\section{HAL Id: halshs-01240093 \\ https://shs.hal.science/halshs-01240093}

Submitted on 29 Aug 2017

HAL is a multi-disciplinary open access archive for the deposit and dissemination of scientific research documents, whether they are published or not. The documents may come from teaching and research institutions in France or abroad, or from public or private research centers.
L'archive ouverte pluridisciplinaire HAL, est destinée au dépôt et à la diffusion de documents scientifiques de niveau recherche, publiés ou non, émanant des établissements d'enseignement et de recherche français ou étrangers, des laboratoires publics ou privés. 


\section{INTERVENIR EN EPS POUR DÉVELOPPER L'ENTRETIEN DE SOI. UNE ÉTUDE DE CAS D'UN ENSEIGNANT EXPÉRIMENTÉ QUI DÉBUTE}

Denis Loizon

Armand Colin | « Carrefours de l'éducation »

$2015 / 2 n^{\circ} 40 \mid$ pages 51 à 65

ISSN 1262-3490

ISBN 9782200930042

Article disponible en ligne à l'adresse :

http://www.cairn.info/revue-carrefours-de-l-education-2015-2-page-51.htm

\section{!Pour citer cet article :}

Denis Loizon, « Intervenir en EPS pour développer l'entretien de soi. Une étude de cas d'un enseignant expérimenté qui débute », Carrefours de l'éducation 2015/2 (n 40), p. 51-65. DOI 10.3917/cdle.040.0051

Distribution électronique Cairn.info pour Armand Colin.

(C) Armand Colin. Tous droits réservés pour tous pays.

La reproduction ou représentation de cet article, notamment par photocopie, n'est autorisée que dans les limites des conditions générales d'utilisation du site ou, le cas échéant, des conditions générales de la licence souscrite par votre établissement. Toute autre reproduction ou représentation, en tout ou partie, sous quelque forme et de quelque manière que ce soit, est interdite sauf accord préalable et écrit de l'éditeur, en dehors des cas prévus par la législation en vigueur en France. Il est précisé que son stockage dans une base de données est également interdit. 


\section{Denis Loizon}

Denis.Loizon@wanadoo.fr

\section{INTERVENIR EN EPS \\ POUR DÉVELOPPER \\ L'ENTRETIEN DE SOI.}

UNE ÉTUDE DE CAS

D'UN ENSEIGNANT

EXPÉRIMENTÉ QUI DÉBUTE

Le but de cet article est de montrer le poids des déterminants personnels dans les décisions didactiques des enseignants, avant et pendant l'action, même si celles-ci sont également influencées par d'autres déterminants comme le public auquel s'adresse l'enseignant ou les textes institutionnels (Goigoux, 2007). Pour réaliser cette démonstration, nous prenons appui sur la notion de filtres de l'action didactique (Loizon, 2009) identifiés au travers du « déjà-là expérientiel » et du « déjà-là conceptuel » de l'enseignant dans le champ de la didactique clinique (Carnus, Terrisse, 2013). Après avoir présenté le cadre théorique et méthodologique de ce champ scientifique en émergence dans les Sciences de l'intervention en EPS et en sport (Musard, Loquet, Carlier, 2010), nous détaillerons cette notion de filtre de l'action didactique car ces filtres participent aux prises de décisions pas toujours conscientes des enseignants d'EPS avant et pendant l'action didactique. Nous faisons l'hypothèse suivante: connaître les filtres du sujet enseignant permettrait d'expliquer une part de son activité didactique souvent opaque au sujet lui-même.

\section{CADRE THÉORIQUE ET MÉTHODOLOGIQUE DE LA DIDACTIQUE CLINIQUE}

Nos options théoriques et méthodologiques s'inscrivent dans le champ de la didactique clinique (Terrisse, Carnus, 2009) qui postule que la prise en compte du sujet enseignant devient un élément incontournable pour comprendre certains 
faits didactiques car les concepts de la didactique ne peuvent pas tout expliquer. Certains auteurs (Buznic-Bourgeacq et al., 2010 ; Carnus, 2003, 2009; Loizon, 2004 ; Terrisse, 2008) ont bien montré au travers d'études de cas en EPS que certains savoirs étaient enseignés ou que certaines actions étaient réalisées à l'insu des enseignants. Ce cadre théorique s'appuie donc sur les concepts de la didactique, mais aussi sur une dimension plus clinique sans visée de soin (Yelnik, 2005). Cette étude se centre donc sur le sujet enseignant en s’appuyant sur une théorie du sujet d'inspiration psychanalytique qui affirme une consistance propre du psychisme par rapport à l'organisme et au social, et qui s'intéresse au sujet singulier en situation comme le rappellent Beillerot et al. (1996). En didactique clinique, cette approche repose sur trois postulats:

- Le sujet est singulier. Cette singularité est à mettre en relation avec son histoire personnelle et professionnelle, son rapport à l'activité physique...

- Le sujet est divisé entre ce qu'il fait et ce qu'il aimerait faire, entre ce qu'il dit et ce qu'il fait. Cette division est repérable dans les tensions ou les dilemmes qu'il évoque dans les entretiens.

- Le sujet est toujours assujetti à un contexte, ici le contexte scolaire avec son cadre institutionnel, les programmes, mais aussi une certaine culture scolaire qui traduit son rapport institutionnel au savoir (Chevallard, 1989).

L'énoncé de ces différents postulats a des répercussions au plan méthodologique. La nécessité de recueillir le discours de l'enseignant en situation d'enseignement « ordinaire » relève de la première condition de ces recherches en didactique clinique. Dans ce discours, l'analyse porte en priorité sur les objets d'enseignement qui sont enseignés (dimension didactique), mais elle porte aussi sur la dimension clinique avec « les récurrences, les répétitions et l'insistance des signifiants, notamment en y faisant apparaître ce qui insiste au-delà de ce qui y est énoncé, autant que les ambiguités, les contradictions et l'équivoque des termes utilisés (ce qui passe à l'insu du sujet parlant) » (Terrisse, 2000, p. 99). La deuxième condition renvoie à l'après-coup, c'est-à-dire au temps où le sujet va procéder à une réélaboration, à un remaniement de son discours dans les entretiens post-séance et dans l'entretien d'après-coup. Enfin, la troisième condition suppose une formalisation des dires du sujet; ses paroles rendent compte alors de sa position subjective.

Dans la leçon d'EPS, le professeur est amené à prendre des décisions nombreuses, à faire des choix en fonction de certains déterminants objectifs (les élèves et les textes officiels) ou subjectifs (Goigoux, 2007). II transforme alors les savoirs à enseigner dictés par les programmes scolaires en savoirs enseignés comme le rappelle Chevallard (1991) lorsqu'il décrit les mécanismes de la transposition didactique interne. Les « déjà-là » de l'enseignant fonctionnent alors comme des « filtres » car ils modifient les savoirs et influencent les décisions didactiques liées aux savoirs à enseigner comme l'a montré Loizon (2009, 2013). Dans une visée modélisante, cet auteur a identifié dans les déclarations des enseignants d'EPS 
étudiés, quatre « déjà-là », du plus explicite au plus implicite qui filtrent ainsi les décisions didactiques (Carnus, 2003):

- Le « déjà-là intentionnel spécifique » correspond aux intentions didactiques se rapportant spécifiquement à la leçon observée avec ses objectifs spécifiques. II est clairement identifié dans les entretiens ante ou post séance.

- Le « déjà-là intentionnel général » est lui aussi repérable dans les entretiens ante et post séance quand les enseignants expliquent leurs intentions, les objectifs généraux du cycle en EPS pour justifier les choix opérés pour les leçons.

- Le « déjà-là conceptuel » renvoie aux conceptions de l'enseignant, c'est-à-dire aux éléments qui structurent en profondeur ses intentions didactiques (Durand, 1996). Plus difficile à mettre à jour, le chercheur le repère dans les récurrences, dans ce qui relève des représentations, des croyances et des valeurs du sujet dans les entretiens d'accès au « déjà-là ».

- Le « déjà-là expérientiel » est identifié très clairement à travers les expériences diverses concernant leurs pratiques sportives relatées par les professeurs d'EPS dans les entretiens d'accès au « déjà-là ».

Dans les déclarations des sujets lors des entretiens, le repérage des différents « déjà-là » n'est pas systématiquement lié à un type d'entretien. Il arrive en effet que le sujet évoque ses intentions spécifiques dans l'entretien d'accès au « déjà-là » ou même qu'il raconte un pan de son histoire de pratiquant dans les entretiens ante ou post (Loizon, Carnus, 2014). Le « déjà-là conceptuel » est repéré aujourd’hui par les chercheurs au travers de ses différents « rapports à... »: rapport à l'activité physique, rapport au corps, rapport à l'autre, rapport à l'enseignement (Loizon et al., 2013; Maizières, 2011; Simondi, Perrenoud, 2011).

Ces « déjà-là » s'inscrivent sur un axe chronologique englobant tout ce qui s'est passé avant les observations du chercheur ; ils concernent donc le sujet et son histoire; ils expriment toute la singularité du sujet enseignant. Ces quatre déjà-là agissent donc comme des filtres personnels qui influencent fortement le choix des savoirs à enseigner (les intentions didactiques), mais aussi les décisions didactiques prises dans la leçon (Loizon, Carnus, 2012, 2014). Il existerait une relation de causalité entre ces déjà-là : l'expérience des sujets contribue à structurer leurs conceptions qui elles-mêmes vont influencer les intentions didactiques (générales et spécifiques) et les prises de décisions didactiques. On peut comprendre ainsi l'effet de la transposition didactique interne: deux enseignants qui doivent enseigner les mêmes savoirs n'enseigneront jamais réellement ces mêmes savoirs car ils ont des filtres, des déjà-là, fondamentalement différents à cause de leur histoire personnelle. C'est cette influence de l'expérience personnelle de pratiquant que nous allons tenter de montrer à partir de l'étude de cas de cette professeure d'EPS qui débute dans l'enseignement du step et de la musculation. 
Dans le cadre des études exploratoires à visée descriptive et compréhensive, nous utilisons la méthode du cas par cas comme méthode de recherche (Albarello, 2011). Dans cet article, nous présenterons une seule étude de cas réalisée auprès d'une femme, professeur d'EPS expérimentée (12 ans d'enseignement de l'EPS), qui enseigne en lycée général et technique depuis 9 ans. Cette étude porte sur une professeure d'EPS confrontée à l'enseignement de nouvelles activités physiques et sportives regroupées dans la compétence propre « entretien de soi » (CP5). Le step et la musculation sont deux activités qui sont nouvelles pour elle car elle ne les a jamais enseignées. II s'agit de son premier cycle avec une classe de seconde dans ces deux activités inscrites aujourd'hui dans les programmes de la CP5 (Programmes EPS, enseignement commun, 2010).

L'objectif initial de cette recherche est d'identifier les savoirs qui font l'objet d'un enseignement réel aux élèves à travers un corpus principal composé de la retranscription verbatim des séances filmées. Pour comprendre les choix réalisés par cette enseignante, nous nous appuyons sur le corpus secondaire qui prend en compte ses déclarations lors des divers entretiens. Pour cela, nous utilisons une méthodologie spécifique en didactique clinique en relation avec les trois temps de l'action didactique préconisés par Terrisse et Carnus (2009):

- Un premier temps pour recueillir les « déjà-là » du sujet sous ses différentes formes qui président aux décisions didactiques (planification et réalisation de l'action) sous forme d'un entretien d'accès au « déjà-là » (Ent Dj). Dans cet entretien, le sujet est questionné sur son expérience professionnelle et personnelle en lien avec les activités physiques qu'il a pratiquées ou pratique encore (déjà-là expérientiel), sur ses conceptions de l'EPS (« déjà-là conceptuel ») ainsi que sur ses intentions didactiques relatives au cycle qu'il met en place, ici le step et la musculation. Toujours en amont des observations, un entretien ante séance, différent de l'entretien d'accès au « déjà-là », précède chaque observation de séance afin d'obtenir des informations sur ce qui va être enseigné pour accéder aux intentions didactiques spécifiques.

- Un deuxième temps, celui de l'épreuve qui constitue le moment de vérité où le sujet est convoqué face à la classe (Terrisse, 2000); c'est toute l'activité de l'enseignante que nous enregistrons en vidéo. Trois leçons d'EPS de deux heures sont filmées sur les cinq qui composent ce cycle consacré au step et à la musculation. Le temps réel d'enseignement est estimé à 1 h 30 compte tenu du déplacement et des temps de déshabillage et de rhabillage des élèves. Le discours de l'enseignante durant ces trois leçons est retranscrit pour pouvoir procéder à une analyse de contenu (Bardin, 1998) visant à identifier les objets d'enseignement en grandes catégories. Nous ne rentrerons pas dans le détail de ce qui est effectivement enseigné.

- Le troisième temps est celui de l'après-coup, ce que Lacan (1966) nomme le « temps pour comprendre » ou encore «le temps où ça se signifie » avec un retour sur l'action. L'après-coup est alors un moyen utilisé par le chercheur 
pour accéder au point de vue du sujet, à ses interprétations, à toute sa subjectivité prise dans son fonctionnement didactique. Pour cela, nous utilisons deux types d'entretiens: des entretiens semi-directifs plus classiques dits entretiens post séance (Ent post) qui consistent à recueillir des informations sur ce qui a été enseigné, bien souvent juste après les leçons observées, et un entretien d'après-coup $(A C)$ réalisé après une première analyse des résultats. Nous recherchons dans ces entretiens toutes les récurrences, ce qui insiste parfois à l'insu du sujet.

Ce dernier entretien est très important pour le chercheur car il lui permet de confronter ses propres interprétations avec celles du sujet. De même, pour limiter les effets de surinterprétation du chercheur, nous sollicitons un autre membre du groupe de chercheurs en didactique clinique pour vérifier si nos interprétations peuvent être fiables comme le préconise Terrisse (2014) car « le chercheur est aussi un sujet doté d'un inconscient ». Enfin, avant toute publication, nous envoyons le texte de l'article au sujet enseignant avec tous les verbatim et nous tenons compte de ses remarques éventuelles.

Dans cette visée compréhensive de l'activité didactique de cette enseignante, nous procédons donc à quatre types d'entretiens qui vont composer notre corpus secondaire: un entretien d'accès aux « déjà-là » (Ent Dj), puis des entretiens ante (Ent ante) avant chaque leçon filmée, puis des enregistrements vidéos des séances d'EPS en situation ordinaire, puis des entretiens après les leçons filmées (Ent post), et enfin un entretien d'après-coup (AC) mené plusieurs semaines après les observations pour revenir sur des points très spécifiques qui méritent de nouvelles explications de la part du sujet afin de mettre ainsi à jour la logique qui anime l'enseignante prise dans le didactique.

Notre objet d'étude pour cet article concerne les choix didactiques de l'enseignant quand celui-ci ne connaît pas bien l'activité enseignée comme c'est le cas pour cette enseignante. II s'agit ici de comprendre toute la subjectivité de l'enseignante à travers ses décisions didactiques : leurs origines, les choix réalisés, les tensions vécues, les satisfactions ou encore les difficultés vécues... Nous nous intéresserons également à la manière dont s'y prend le sujet pour conserver son statut de « sujet supposé savoir » dans ce cas très précis.

Pour présenter nos résultats sous forme de vignette didactique clinique, nous utiliserons les trois temps de la méthodologie didactique clinique présentée plus haut.

\section{LES « DÉJÀ-LÀ » DE L'ENSEIGNANTE}

La mise en évidence des différents « déjà-là » se réalise à partir de l'analyse des déclarations de l'enseignante lors des différents entretiens même si le premier entretien que nous qualifions d'accès au « déjà-là » apporte de nombreux indices. 
Comme nous l'avons déjà évoqué, nous recherchons surtout dans ses paroles ce qui est récurrent, ce qui « signifie » pour le sujet (Lacan, 1977) et cela peut apparaître dans n'importe quel entretien.

Lors de l'entretien de « déjà-là » (Ent Dj) réalisé quelques semaines avant de commencer les enregistrements vidéo, nous questionnons l'enseignante sur son parcours professionnel. Elle nous raconte alors qu'elle a fait des études en STAPS pour devenir enseignante et satisfaire deux envies: " je voulais faire du sport, me dépenser... j'aime ça. (...) Et puis m'occuper d'enfants » (Ent Dj). Pendant ses études universitaires, elle fait la découverte d'une toute nouvelle activité pour elle : la course d'orientation (CO) : "pendant mes études en Staps, je découvre la CO. C'était génial... un plaisir de découvrir, de réussir » (Ent Dj). Tout au long du cycle de CO qu'elle suit avec le formateur, elle se passionne de plus en plus car elle découvre une certaine liberté dans le choix des trajets, rien ne lui est imposé, sauf les postes avec les balises. À elle de se débrouiller pour les trouver: «je me sentais libre !!! Un sentiment tout nouveau pour moi ».

Ce plaisir éprouvé au plus profond d'elle-même lui donne envie de faire connaître la CO à l'occasion de ses mutations professionnelles dans plusieurs établissements, une envie de faire partager son plaisir : "dans tous les établissements où je suis passée, je m'organisais pour faire un cycle de $\mathrm{CO}$, même à l'intérieur de l'établissement... je voulais faire vivre cela à mes élèves, pour qu'eux aussi ils se sentent libres, même à l'intérieur des murs de l'école ». Elle construit de nombreuses cartes, d'abord de manière très simple, puis elle va se perfectionner pour devenir une vraie spécialiste de cartographie en utilisant des logiciels spécifiques.

De coureur expérimenté, elle va devenir peu à peu une vraie spécialiste passionnée en passant de nombreux diplômes fédéraux : "j'ai passé les brevets d'états pour pouvoir former des jeunes, former des enseignants... » Elle réussit alors à conjuguer ses deux passions : I'enseignement pour "s'occuper d'enfants de tous les âges », et son besoin de dépense physique tout en alliant une forte dimension cognitive: "il ne faut pas se tromper dans la lecture; le moindre petit indice est très important. La CO, c'est du souffle et de la réflexion !!! »

Au-delà de sa passion pour l'enseignement, et toujours pour la faire partager, elle s'engage dans la vie associative au plus haut niveau régional pour participer au développement régional de la course d'orientation: "Je suis aujourd'hui présidente de la ligue de $\mathrm{CO} »$. Une passion qui se traduit par un engagement important tant en formation qu'en responsabilité au niveau associatif.

À travers ce parcours rapidement brossé, nous comprenons comment la découverte d'une activité sportive pousse le sujet à s'investir dans différents domaines qui mêlent à la fois les sphères personnelles et professionnelles. Le poids du « déjà-là expérientiel » s'avère déterminant dans l'engagement de cette jeune enseignante. Comment ces différentes expériences vont-elles structurer ses conceptions (son « déjà-là conceptuel ») ? 
En effet, cette professeure expérimentée essaie de transmettre son expérience, mais aussi son rapport au savoir. Cette volonté de transmettre son savoir personnel appelée « conversion didactique » en didactique clinique (Buznic-Bourgeacq et al., 2010) correspond à une conversion de sa propre expérience corporelle ; elle est particulièrement forte dans ses propos: "je voulais que mes élèves vivent le plaisir de trouver la balise... un peu comme une chasse au trésor, mais avec un engagement physique très fort... et une réflexion, une stratégie pour trouver le plus vite possible, sans perdre de temps » (AC).

Dans cet entretien, on relève aussi la trace d'un sujet très divisé entre ce qu'il aimerait faire et ce qu'il réussit à faire avec ses élèves : " quand je n'arrive pas à faire partager mon plaisir... quand les élèves résistent... qu'ils ne s'investissent pas assez.» La déception, la frustration sont grandes quand les élèves ne rentrent pas dans l'activité, quand l'enseignante n'arrive pas à faire partager les émotions qu'elle a ressenties en pratiquant cette activité.

À partir de ce qu'elle nous livre dans cet entretien de « déjà-là », il est possible de dire que son « déjà-là conceptuel » défini comme un système de croyances, de valeurs, de représentations propres au sujet, peut s'organiser autour de quelques idées fortes récurrentes dans ses déclarations aussi bien dans les entretiens ante et post que dans l'entretien d'après coup comme:

- l'engagement physique: «ce que je recherche en priorité en EPS, c'est l'investissement physique, l'engagement des élèves »;

- le plaisir dans l'effort: "le plaisir, il passe par la réussite de l'action... la satisfaction d'avoir été au bout de soi, au bout de l'effort »;

- la compétition : " ce que j'aime aussi, c'est la compétition, ça t’oblige à aller chercher dans tes ressources si tu veux gagner... » (Ent Dj).

On pourrait résumer ses conceptions autour de quatre mots, de quatre éléments forts de son épistémologie personnelle (Brousseau, 1998) qui reviennent souvent dans les différents entretiens que nous avons eus avec cette enseignante : investissement physique, effort, compétition et plaisir. C'est ce noyau dur qui constitue selon nous, son « déjà-là conceptuel »; il fait partie de son identité professionnelle (Perez-Roux, 2011). II correspond à son rapport à l'activité course d'orientation et à une forme de rapport à soi (Loizon et al., 2013; Maizières, 2011).

Ce noyau dur est présent dans le premier entretien ante séance lorsqu'elle nous annonce ses objectifs du cycle, c'est-à-dire son « déjà-là intentionnel », ses intentions didactiques générales sur le cycle de step: «Sur le step, l'objectif, il est essentiellement de travailler sur le rythme cardiaque, la coordination du haut, du bas du corps, la mémorisation d'un enchaînement que je leur impose sur une musique donnée... ils sont là juste pour reproduire et refaire ce qu'on leur demande (...) ce que je veux avant tout, c'est qu'ils s'engagent ». La priorité, c'est l'engagement physique à travers un travail important sur le rythme cardiaque qui exprime à la fois un rapport à soi et à l'activité, signes de son identité professionnelle. 
Concernant ses intentions didactiques pour le cycle de musculation, nous retrouvons là encore des éléments forts de ses conceptions comme la notion d'effort qui est primordiale pour elle: «surtout travailler sur la notion d'effort en musculation. C'est-à-dire tenir un effort dans le temps, que ce soit un effort de concentration, pour le step, donc la mémorisation et la concentration et de concentration aussi pendant le mouvement de muscu pour pas dénaturer le mouvement ».

À côté de ces éléments nodaux, on retrouve d'autres objectifs plus traditionnels comme les mouvements, les positions: «en musculation, je vais mattacher davantage à être très exigeante sur le mouvement, la position du corps qu'ils respectent vraiment les positions sécuritaires, qu'ils aient un comportement d'entraide par rapport à celui qui travaille notamment quand les charges vont augmenter. Je ne vais pas forcément travailler sur des rapports poids/puissance parce que de toute façon j'ai pas le matériel qui me permet de travailler là-dessus et puis, je pense que par rapport à des secondes, cet objectif, il n'est pas forcément vital. S'ils sont déjà là à faire correctement le mouvement, et à travailler le bon muscle, se connaître corporellement, savoir ce qu'ils travaillent, voilà ce sera déjà bien ». Ces objectifs de l'enseignante présentés comme des intentions générales nous semblent avoir moins d'importance à ses yeux car elle y revient peu dans les différents entretiens à la différence des éléments de son « déjà-là conceptuel » qui sont présents sous des formes différentes dans les différents entretiens.

Les entretiens nous ont donc permis de mettre en évidence le « déjà-là » de cette enseignante expérimentée, et plus particulièrement son « déjà-là conceptuel » qui se traduit dans son rapport à l'enseignement, mais comment celui-ci filtre-t-il son activité didactique face à la classe durant l'épreuve d'enseignement?

\section{L'ÉPREUVE DE LA SITUATION D'ENSEIGNEMENT}

La situation d'enseignement représente l'épreuve, le temps du réel avec sa contingence selon Terrisse (2008). L'enseignement de ces deux activités, step et musculation, relève d'une véritable épreuve subjective au sens de difficulté (Maleyrot, 2013) pour cette enseignante; cela est perceptible sur plusieurs registres.

Cet enseignement la place dans une position particulière comme souvent chez les enseignants débutants; elle se retrouve dans la position du sujet supposé savoir qui ne sait pas (Ben Jomâa, Terrisse, 2011): "Ben oui moi, le step, c'est pas une activité dans laquelle je suis à l'aise, donc même pas du tout. Donc je pense que l'image que je vais véhiculer auprès des élèves; si déjà moi, je suis pas à l'aise, je ne vais pas les mettre à l'aise [...] donc ça fait beaucoup d'investissement, de ma personne pour que ça se passe le mieux possible pour eux aussi après » (Ent ante 1). Cette caractéristique du sujet qui ne connaît pas l'activité enseignée la contraint à s'entraîner, à répéter les enchaînements de step à la maison : elle se prépare à l'épreuve d'enseignement. À la fin de la première séance, elle nous confie: «Donc 
il ne fallait pas que je me trompe, fallait que... bien que voilà... parce que moi j'avais répété encore plus le matin, donc je commence à la connaître un petit peu la choré, donc je me sens mieux » (Ent post 1).

Contrairement à d'autres activités sportives, l'enseignement du step présente une spécificité: le professeur est obligé de pratiquer, de faire devant les élèves, de montrer systématiquement toute la chorégraphie afin que les élèves puissent la répéter seuls ensuite: "Donc moi je suis obligée de faire les mouvements dans lesquels je suis pas à l'aise forcement, il faut que je compte en même temps, il faut que je me rappelle de la choré, donc ça fait beaucoup d'investissement ». Dans cet extrait, on perçoit l'investissement nécessaire et les difficultés vécues car le professeur qui est obligé de compter, de donner des indications à ses élèves, mais le plus difficile semble être le fait de ne pas voir les élèves car elle est placée devant le groupe avec ses élèves dans le dos. Dans cette situation particulière où elle ne maîtrise pas le savoir, elle est contrainte de piloter la classe sans voir les élèves: «Bon dans l'ensemble, même si je leur tournais le dos, quand je me retournais, j'avais plutôt l'impression que la majorité du groupe, elle était synchro... au niveau mémo, y'a pas trop de décalage donc je pense qu'ils ont bien anticipé chaque petite séquence, donc là c'est gagné (geste de victoire) » (Ent post 1). Cette position extraordinaire I'oblige donc à faire de nombreuses démonstrations car sa préoccupation centrale reste focalisée sur l'engagement physique au cœur de son « déjà-là conceptuel »: "j'essaye qu'ils restent toujours dans l'action, après une démonstration qu'ils reprennent le même mouvement. Donc ça passe par la démonstration » (Ent post 1).

Pour atteindre cet objectif très personnel, en musculation, elle s'y prend complètement différemment en laissant les élèves autonomes: «Pendant qu'il y en a un qui travaille avec le matériel, l'autre qui n'a pas le matériel sollicite un autre groupe musculaire sur un mouvement simple sans matériel » (Ent post 1). Ceci l'amène aussi à faire des propositions innovantes en step par rapport aux programmes d'EPS en lycée : " aujourd'hui pour le step, j'ai prévu alors que c'est pas du tout ce qu'on demande de faire en seconde, je leur demande de créer eux-mêmes un petit enchaînement de seize mouvements. Et puis en plus je pense que si c'est eux qui les choisissent, ils vont se mettre dans une position un peu plus facilitante et du coup ils seront peut-être mieux coordonnés; donc là j'ai prévu un petit travail en sous-groupe, voilà pour voir un petit peu ce que ça va donner... » (Ent ante 3).

Son « déjà-là conceptuel » filtre aussi ses observations, il les guide. Cela se traduit par la mise en évidence de ce que nous appelons en didactique clinique, l'impossible à supporter en référence à Lacan (1977), qui se manifeste dans son cours: "Au niveau, de la fréquence de travail, il y avait beaucoup de temps d'attentes, ce que je ne supporte pas, donc au niveau des récupérations et du travail, c'était pas génial, parce qu'ils faisaient un exercice, puis il y avait 5 minutes qui s'écoulaient et puis ils faisaient le deuxième » (Ent post 3). Elle ne supporte pas les élèves qui sont en situation d'attente, qui ne font pas d'effort. Cet écart entre ce 
qu'elle aimerait en termes de fonctionnement et l'activité perçue des élèves qui attendent, est révélateur de la division structurelle du sujet.

Le « déjà-là » participe à la planification des leçons; il filtre aussi les observations de l'enseignante. Enfin, il intervient également dans les interactions que le sujet entretient avec ses élèves. Durant les leçons consacrées au step, nous relevons dans le verbatim de nombreux feed-backs positifs qu'elle renvoie aux élèves quand ceux-ci s'engagent dans l'activité. Elle s'adresse soit à des petits groupes: " c'est très bien ce que vous faites » (Leçon 2), soit à des élèves particuliers : " c'est très bien ce que tu fais Jessica » (Leçon 1) ou encore «tu t'investis beaucoup aujourd'hui Karl » (leçon 2). Par contre, à aucun moment elle ne revient sur ses interactions au cours des entretiens post leçon.

L'identification des « déjà-là » et l'analyse de la situation d'enseignement mettent en évidence les choix didactiques particuliers de cette enseignante en relation avec son expérience professionnelle quasi inexistante face à ces nouvelles activités. Entre développement de la dimension essentiellement motrice de l'EPS et développement de l'éducation à la santé au travers de la connaissance de soi que l'élève peut acquérir en step et en musculation, la division du sujet enseignant apparaît, notamment au travers de son rapport au savoir, même si ces deux objectifs ne sont pourtant pas contradictoires. L'enseignante s'organise pour conserver son statut de sujet supposé savoir malgré son inexpérience: elle se prépare, répète les chorégraphies en step pour montrer qu'elle sait, alors qu'en musculation, elle préfère ne pas intervenir. Sa méconnaissance des deux activités l'amène à trouver une stratégie particulière pour enseigner le step : elle laisse les élèves créer des enchaînements en step ce qui les maintient dans l'activité à sa grande satisfaction, par contre, à son grand regret, elle n'a pas trouvé d'autre moyen en musculation pour limiter les temps d'attente que de proposer des exercices simples mais pas sur le même groupe musculaire.

Enfin, l'entretien d'après-coup lui permet de revenir sur certains points déjà soulevés dans nos observations comme nous allons le voir.

\section{L'APRÈS-COUP DE L'ENSEIGNANTE}

Dans cet entretien mené deux mois après les séances observées, l'enseignante revient sur les objectifs qu'elle pense avoir atteints: " par rapport au step, c'est l'engagement physique, la confiance en soi par rapport au mouvement et la réalisation du mouvement devant tout le monde. Donc ça je pense que c'est... ils ont quand même pris confiance en eux pour pouvoir le réaliser correctement (...) au niveau de l'aspect concentration, on a une concentration qui apparaît mais plutôt une concentration de court terme, c'est-à-dire juste au moment de la réalisation et à condition qu'il n'y ait pas d'erreur ». Dans son discours, nous retrouvons des éléments forts comme l'engagement physique, mais elle nous donne un autre objectif qu'elle 
n’avait pas énoncé lors des entretiens ante-séance comme le développement de la confiance en soi sur lequel elle insiste fortement à plusieurs reprises au cours de ce dernier entretien. Ce temps de remaniement conduit le sujet à revisiter son activité didactique, à redéfinir de nouveaux objectifs ou simplement à mettre en évidence des intentions opaques chez le sujet qui surgissent à l'occasion de cet entretien.

L'enseignante revient également sur le plaisir perçu chez les élèves, notion fondamentale pour elle comme nous l'avons vu dans son déjà-là conceptuel : "j'ai une satisfaction parce que je pense qu'eux finalement, ils ont réussi à se faire plaisir sur certaines choses, donc à partir du moment où ils ont aussi trouvé du plaisir, je pense que... bon le travail, il reste positif. Heu bon après, c'est vraiment des cycles courts donc on vise des choses faciles ». On voit combien son plaisir est lié au fait que ses élèves éprouvent eux aussi du plaisir.

Si elle est heureuse face à certains comportements observés, elle est déçue par rapport à ses conceptions qui touchent l'investissement des garçons: "le comportement des garçons était assez décevant parce que c'est des garçons qui physiquement ont les moyens de faire des choses très bien et en musculation j'ai été très très déçue de leur investissement... ce sont des gamins qui auraient dû se surpasser, se faire de la compétition entre eux pour essayer vraiment d'être meilleur que l'autre... » (AC). Ces garçons ne sont pas dans le même rapport à l'activité que l'enseignante, ce qui produit une grande déception chez elle.

En fin d'entretien, elle nous fait part de l'évolution de certaines de ses représentations: "j'ai trouvé que les filles étaient beaucoup plus investies, plus sérieuses sur la manière de réaliser les choses, alors que je ne m'attendais vraiment pas à ça. Pour moi, la musculation c'est un truc de garçon, ils vont rentrer tout de suite dedans, ils vont vouloir mettre des charges tout de suite très lourdes... et puis en fait, pas du tout » (AC).

Ce dernier entretien permet donc au professeur de revenir sur son expérience lors de ce cycle. Nous constatons que ses satisfactions et ses déceptions sont très liées à ses conceptions, à son « déjà-là conceptuel », structuré à partir de son rapport personnel à l'activité course d'orientation, sa spécialité sportive, qui déteint (Ben Jomâ, Terrisse, 2011) dans son traitement didactique des activités step et musculation. Si ses représentations de l'investissement filles/garçons se transforment à l'issue du cycle, il semble que le noyau dur de son « déjà-là conceptuel » reste constant, même confronté à l'épreuve de l'enseignement.

\section{CONCLUSION}

L'étude menée auprès de cette enseignante sous la forme d'une étude de cas, même si elle ne peut être généralisable, nous permet de dire que ses « déjà-là » qui constituent son identité de professeur et qui relèvent de son épistémologie 
(Brousseau, 1998), participent activement à ses observations, à ses prises de décisions, à ses satisfactions ou à ses déceptions. Ils déterminent une part importante de son action didactique selon ses propres déclarations. Combinés aux déterminants « public » et « institutionnel » qui n’apparaissent pratiquement pas dans le discours du professeur, ces différents « déterminants personnels » (Goigoux, 2007) participent ainsi à la redéfinition de la tâche de l'enseignante sous forme d'auto-prescription de manière implicite. Ils agissent avant l'action dans les choix didactiques, et pendant l'action en guidant les observations et en influençant certaines des interactions didactiques en direction des groupes ou de certains élèves. Au même titre que les déterminants objectifs (durée du cycle et des séances, conditions matérielles...) sur lesquels l'enseignante fait peu de remarque dans les entretiens, les déterminants personnels du sujet constitués de ses « déjà-là », et plus particulièrement de son rapport au savoir (Beillerot et al., 1996) repéré à travers l'expression de son rapport à l'activité physique, à soi et à l'enseignement (Simondi, Perrenoud, 2011), méritent donc d'être pris en compte dans le cadre des recherches sur l'intervention pour comprendre et expliquer les décisions didactiques du sujet enseignant.

À côté des savoirs enseignés qui relèvent de la dimension motrice, du savoir s'entraîner et de certaines compétences psychosociales, le rapport au savoir de l'enseignant est un élément important qui est transmis à son insu de manière très implicite; nous avons vu combien il générait déception ou plaisir.

Cette étude met en évidence la position symbolique du sujet enseignant face à la difficulté d'enseigner une nouvelle activité comme le step avec ses spécificités, notamment le fait d'avoir des séquences d'enchaînement à démontrer, ce qui nécessite un investissement important de la part du professeur pour conserver son statut de sujet supposé savoir face aux élèves comme chez les enseignants débutants (Buznic-Bourgeacq et al., 2008). On découvre aussi le génie didactique (Mercier et al., 2001) à l'œuvre (les ruses) durant les séances avec la vidéo pour mieux observer l'activité des élèves ainsi que les innovations par rapport aux programmes officiels pour essayer de maintenir la relation didactique et l'investissement des élèves. Au-delà de ces résultats qui montrent le poids important des « déjà-là expérientiel » et « conceptuel » dans l'activité didactique, cette étude de cas interroge le chercheur car au moins deux questions restent sans réponse concernant la prise en compte du contexte et l'effet des entretiens chez le sujet. Curieusement, comme nous l'avons évoqué, les deux autres déterminants (public et institutionnel) qui participent à l'activité enseignante sont peu mentionnés par le sujet: dans ce cas précis d'un premier cycle, de la découverte de nouvelles activités physiques, cela relève-t-il d'un allant de soi, d'une évidence qui mériterait d'être interrogée, ou bien ces déterminants sont-ils secondaires par rapport au déterminant personnel ? Ensuite, même si les entretiens ont pour but premier d'obtenir des réponses aux questions posées par le chercheur, il n'en demeure pas moins que l'enseignant, engagé dans ce travail réflexif avec l'aide du 
chercheur, en ressort quelque peu transformé car tout un pan de son activité lui apparaît plus clairement comme l'évoque un autre enseignant avec lequel nous avons travaillé de manière assez similaire dans le cadre d'une autre recherche en didactique clinique: "Grâce à cet échange avec le chercheur, j'ai l'impression d'avoir franchi une marche, un cap sur la compréhension de ma façon d'enseigner qui était très intuitive et qui, maintenant est plus cohérente à travers ce qui m’a le plus marqué, notamment mon vécu de combattant, mon « déjà-là expérientiel » pour reprendre le terme employé dans cette recherche » (Loizon, Martin, 2006, p. 116).

Denis Loizon

Laboratoire IREDU (ÉA 7389)

Université de Bourgogne Franche-Comté

\section{Bibliographie}

Albarello L. (2011). Choisir l'étude de cas comme méthode de recherche. Bruxelles: De Boeck Université.

Bardin L. (1998). L'analyse de contenu. Paris: PUF.

Beillerot J., Blanchard-Laville C., Mosconi N. (1996). Pour une clinique du rapport au savoir. Paris: L'Harmattan.

Ben Jomâa H., Terrisse A. (2011). De l'expertise à l'enseignement : analyse comparative du rapport au savoir de deux enseignants d'EPS de spécialités sportives différente. Éducation \& Didactique, $\mathrm{Vol} 5, \mathrm{n}^{\circ} 3, \mathrm{p} .61-80$.

Brousseau G. (1998). Théorie des situations didactiques, Recherches en Didactique des Mathématiques. Grenoble: La Pensée sauvage.

Buznic-Bourgeacq P., Terrisse A., Lestel G. (2008). Expérience personnelle et expérience professionnelle dans l'enseignement de l'EPS: deux études de cas contrastées en didactique clinique. Éducation \& Didactique, Vol 2, n³ 3 p. 77-95.

Buznic-Bourgeacq P., Terrisse A., Margnes E. (2010). La transmission du savoir expérientiel en EPS : études de cas et analyses comparatives en didactique Clinique. eJRIEPS 20, p. 26-47. Carnus M.-F. (2003). Analyse didactique du processus décisionnel de l'enseignant d'EPS en gymnastique : une étude de cas croisés. In C. Amade-Escot (éd.). Didactique de l'éducation physique, état des recherches. Paris : Éditions Revue ÉP.S., p. 193-224.

Carnus M.-F. (2009). Pour une didactique clinique de l'EPS. Perspective pour la formation des enseignants. Note de synthèse pour I'habilitation à diriger des recherches. Université Paris Ouest Nanterre la Défense.

Carnus M.-F., Terrisse A. (2013). Didactique clinique de l'EPS. Le sujet enseignant en question. Paris : Éditions ÉP.S.

Chevallard Y. (1989). Le concept de rapport au savoir, rapport personnel, rapport institutionnel, rapport officiel. Actes du séminaire de didactiques des mathématiques et de I'information, Grenoble : LSD Imag et Institut J. Fourier, p. 211-235.

Chevallard Y. (1991). La transposition didactique, du savoir savant au savoir enseigné, avec un exemple d'analyse de la transposition didactique. Grenoble: La Pensée Sauvage. 


\section{INTERVENIR EN EPS POUR DÉVELOPPER L'ENTRETIEN DE SOI}

Durand M. (1996). L'enseignement en milieu scolaire. Paris : PUF.

Goigoux R. (2007). Un modèle d'analyse de l'activité des enseignants. Éducation \& Didactique, Vol 1, $n^{\circ} 3$, p. 47-70.

Lacan J. (1966). Écrits. Paris : Seuil.

Lacan J. (1977). Ouverture de la section clinique. Ornicar, n 9.

Loizon D. (2009). Les filtres personnels dans I'action didactique. In A. Terrisse et M.-F. Carnus (coord.). Didactique clinique de l'éducation physique et sportive (EPS). Quels enjeux de savoirs? Bruxelles: De Boeck, p. 83-100.

Loizon D. (2013). L'enseignant d'EPS et ses filtres déjà-là. In M.-F. Carnus et A. Terrisse (dir.). Didactique clinique de l'EPS. Le sujet enseignant en question. Paris : Éditions ÉP.S., p. 13-21. Loizon D., Carnus, M.-F. (2012). Le déjà-là décisionnel chez les enseignants novices en EPS. Un outil pour comprendre la réalité de leurs enseignements. In G. Carlier, C. Borges, M. Clerx et C. Delens (coord.). Identité professionnelle en éducation physique. Parcours des stagiaires et enseignants novices. Louvain-la-Neuve : Presses Universitaires de Louvain, p. 199-215.

Loizon D., Carnus M.-F. (2014). L'influence des déterminants personnels dans les choix didactiques des enseignants d'EPS. eJRIEPS, 33, p. 30-48.

Loizon D., Charvy N., Cartierre N. (2013). L'accompagnement dans la professionnalisation du métier d'enseignant. Le point de vue des formateurs. Revue Phronésis, Vol 2, n 4 , p. 50-60.

Loizon D., Martin D. (2006). Des savoirs enseignés à l'expérience des enseignants. STAPS, $n^{\circ} 72$, p. 107-118.

Maizières F. (2011). L'Éducation musicale à l'école primaire: quelles relations avec le rapport personnel à la musique de l'enseignant? Les Sciences de l'éducation. Pour l'ère nouvelle, Vol. 44, $n^{\circ} 1$, p. 103-124.

Maleyrot E. (2013). Les professeurs des écoles maîtres formateurs saisis par les épreuves professionnelles. Notes du CREN, $n^{\circ} 16$.

Mercier A., Lemoyne G., Rouchier A. (2001). Le génie didactique. Usages et mésusages des théories de l'enseignement. Bruxelles: De Boeck Université.

Musard M., Loquet M., Carlier G. (2010). Sciences de l'intervention en EPS et en sport. Résultats de recherche et fondements théoriques. Paris : Éditions Revue ÉP.S.

Perez-Roux T. (2011). Identité(s) professionnelle(s) des enseignants. Les professeurs d'EPS entre appartenance et singularité. Paris : Éditions ÉP.S.

Simondi E., Perrenoud B. (2011) Savoirs et éthique dans l'accompagnement. Recherche et Formation, $n^{\circ}$ 66, p. 79-92.

Terrisse A. (2000). Épistémologie de la recherche clinique en sports de combat. In A. Terrisse (dir.). Recherches en Sports de combat et Arts Martiaux, État des lieux. Paris : Éditions revue ÉP.S., p. 95-108.

Terrisse A. (2008). Le sujet en didactique clinique de l'EPS : conditions et conséquences pour la recherche. eJRIEPS ${ }^{\circ} 15$, p. 179-197.

Terrisse, A. (2014). La didactique clinique en EPS : intérêt et enjeux pour la formation des enseignants. In M. Quidou. Innovations théoriques en STAPS et implications pratiques en EPS : Les sciences du sport en mouvement. Paris : L’Harmattan, p. 562-582. 
Terrisse A., Carnus M.-F. (2009). Didactique clinique de l'éducation physique et sportive (EPS). Quels enjeux de savoirs? Bruxelles: De Boeck Université.

Yelnik C. (2005). L'entretien clinique de recherche en sciences de l'éducation. Recherche et Formation, $n^{\circ}$ 50, p. 133-146. 